\title{
IOTAN: LA CUMBRE DEL NUEVO SIGLO
}

Marisa Rodríguez Mojón*

Las dos últimas cumbres que ha celebrado la Organización del Tratado del Atlántico Norte, en Washington (1999), y en Praga (Noviembre de 2002), tenían como objetivo acordar aquellos cambios que permitieran a esta organización de defensa acomodarse a las transformaciones del entorno, tanto las resultantes de la globalización del sistema internacional, como las derivadas del brutal ataque terrorista contra los Estados Unidos que tuvo lugar el 11 de Septiembre de 2001. Globalización entendida como un fenómeno que desborda la simple transformación de las relaciones económicas mundiales, para implicar a la totalidad de las interacciones: políticas, sociales, culturales, económicas, y de seguridad, así como a la velocidad a la que éstas se realizan. Incluyendo la permeabilidad de fronteras, y la compresión de distancias.

La transformación de riesgos y amenazas que se previó al terminar la guerra fría ha dejado de ser un presupuesto teórico después del 11 de Septiembre de 2001. Hasta tal punto, que se ha creado un nuevo escenario, dentro del cual considerar que la eficacia de la OTAN podría mantenerse sin alterar el concepto que la concibió como una "maquinaria de guerra" exclusivamente, se hubiera quedado muy corto.

En la reunión de Washington, en 1999, se decidieron cambios que alteraron su condición de alianza defensiva centrada en el escenario europeo, para transformarla en una alianza de "defensa colectiva", incluyéndose nuevos riesgos, nuevas misiones

* Marisa Rodríguez Mojón es Profesora de Ciencia Política en la Suffolk University, campus de Madrid, colaboradora del CESEDEN y miembro de la Asociación CastellanoManchega de Sociología. 
y una iniciativa de capacidades defensivas, además de concretarse su ampliación al incluir a tres países de los que durante la guerra fría se llamaban del Este y que ahora han retrocedido nuevamente a su anterior condición de centroeuropeos: La República Checa, Polonia y Hungría, lo que ya había sido acordado en la cumbre de la Alianza en Madrid, en 1997. Los dirigentes de la alianza consideraron, también que, para mejorar su eficacia y convertirla en la estructura de seguridad defensora y garante del modo de vida occidental, era necesario conseguir acercar a sus objetivos a todos los países de cultura occidental, para lo cual se aprovechó la reacción de solidaridad rusa ante los ataques terroristas del 11 de Septiembre (similares a los que ese país había sufrido por parte de los chechenos hacía no tanto, y que se repetirían varias veces más) para conseguir un acuerdo con Rusia, que ha permitido la creación del Consejo OTAN-RUSIA. De hecho, durante la cumbre de la OTAN celebrada en Praga, hubo un representante de la Federación Rusa, que se sentó en el Consejo.

La Cumbre de Praga, celebrada a finales del año 2002, ha supuesto un avance importante sobre los cambios introducidos en Washington. Se ha "ampliado la ampliación", incluyendo a otros siete países anteriormente pertenecientes al área del Pacto de Varsovia (Bulgaria, Estonia, Lituania, Letonia, Rumania, Eslovaquia, y Eslovenia), y se ha decidido transformar su estrategia, y su organización militar, de manera que se sentaran las bases de lo que los políticos y los analistas occidentales han denominado "la OTAN del futuro".

\section{UNA PLATAFORMA DE DIALOGO INFORMATIVO}

Poco después de celebrarse esta cumbre, el día 26 de Noviembre de 2002, un grupo de expertos civiles y militares, españoles y estadounidenses, se reunieron en la sede del campus de Madrid de la Universidad de Suffolk (Boston), para conversar sobre las transformaciones estratégicas que habían tenido lugar durante esta cumbre, repasar los acontecimientos que habían llevado a ello, y contribuir a trasladar este tipo de debates al seno de la comunidad intelectual no-militar. También se pretendió crear un ambiente de intercambio de ideas y comunicación, de manera que, por una parte, los estudiantes y profesores tuvieran la ocasión de enterarse del contenido de los debates y acuerdos de la cumbre de la OTAN, y por otra, crear un ambiente de diálogo que permitiera a los representantes de las plataformas políticas oficiales, y del mundo militar, conocer las opiniones de los estudiantes y del mundo académico sobre asuntos que, tradicionalmente, han estado poco abiertos a la difusión entre la sociedad civil. El encuentro fue auspiciado por el Instituto Español de Estudios Estratégicos, del Ministerio de Defensa español.

La reunión se abrió con unas palabras de bienvenida por parte del Decano del campus, Dr. Leslie Croxford, quien dio paso a la profesora de ciencia política del centro, para que leyera un texto consensuado, en el cual se puso de relieve la 
opinión de la comunidad académica del centro sobre tres aspectos de la situación internacional contemporánea:

Primero, la capacidad de los actos terroristas para crear situaciones de angustia de difícil control por parte de las autoridades con los métodos tradicionales, así como para alterar el equilibrio del sistema mundial, dar impulso al comercio internacional de armamentos, y propulsar la fabricación de armas de destrucción masiva.

Segundo, los posibles problemas que se derivarían de una reacción estatal equivocada en la búsqueda de soluciones innovadoras con las que enfrentarse a este nuevo tipo de riesgos. En este sentido, se subrayó el peligro que encerraría el intento de cualquier Estado de asumir una situación hegemónica en la esperanza de que ello le permitiría controlar mejor la situación mundial, y se recordó la importancia del debate abierto y libre para poder identificar los verdaderos problemas implicados en los conflictos de un mundo multicultural, multireligioso, y con muchas ideologías diferentes, de manera que se pudieran implementar las mejores soluciones, en el momento más oportuno, y de la forma más correcta posible.

Por todo ello, y ante los riesgos (no menores) de caer en procesos destructivos de sobre-exposición hegemónica, capaces de crear más confusión que soluciones, se afirmó la importancia de seguir confiando en la capacidad de la Organización de Naciones Unidas como plataforma de debate y resolución de conflictos. Reforzando y reajustando la institución, en caso necesario, pero no saltando por encima de ella.

Tercero, y finalmente, se expuso la esperanza de la comunidad académica de Suffolk, Madrid campus, de que los acuerdos resultantes de la cumbre de la OTAN celebrada en Praga la semana anterior sirvieran para reforzar la confianza de los ciudadanos occidentales cuyos gobiernos son miembros de esa alianza, al incorporar no solamente a nuevos miembros europeos, sino también nuevos mecanismos de actuación que combinarán la capacidad de la misma para enfrentarse a los nuevos riesgos del mundo actual sin interferir con el mantenimiento de un orden mundial "respetuoso y respetable", basado en los principios de la carta de la ONU.

\section{EL DEBATE ACADÉMICO}

En este mismo sentido se expresó, más tarde, el profesor Octavio Uña, quien subrayó el hecho de que los cambios en materia de seguridad internacional no se limitaban a las consecuencias de la globalización, sino que tenían que ver con la pérdida de la racionalidad tradicional. Los acontecimientos del 11 de Septiembre, para el profesor Uña, evidenciaban la búsqueda de otras formas de racionalidad en las relaciones entre los Estados y los pueblos, la resurección de teorías de "la elección divina" (los singulares egregios) de los pueblos y de las elites, con el retorno a una fuerte irracionalidad de los procesos, con la subsiguiente caída de la ideología y su 
reemplazo por la religión como arma de acción. Por ello, el fin de la guerra fría no había sido el fin de la historia, sino el comienzo del fin de la racionalidad. Debido a ello, propuso trabajar en la búsqueda de fórmulas que permitiesen la recuperación de la racionalidad a nivel mundial.

El profesor Antonio Marquina, por su parte, hizo duras críticas a la aparente tendencia de algún Estado por intentar solucionar los problemas del terrorismo atacando a otros Estados, y rompiendo, de esa manera, la legalidad internacional conseguida con mucha dificultad.

Durante las ponencias y debates que se sucedieron a lo largo de la tarde, se pudo apreciar un clima de consenso mayoritario, respecto a la importancia de adecuar los sistemas de seguridad occidentales a los riesgos emergentes en el comienzo del siglo XXI, sin necesidad de proceder a destrucciones gratuitas. Para llegar a esta conclusión, la mayor parte de los ponentes hicieron un breve repaso de la historia de la OTAN, y/o de la evolución de la historia de la seguridad mundial en las últimas décadas. Dentro de esta línea, el coronel Gonzalo Parente destacó el hecho de que la experiencia histórica de la OTAN daba un saldo positivo, en el sentido de que había servido para conseguir que la zona euroatlántica sea hoy "el espacio de mayor seguridad, paz y prosperidad del mundo". Esto se ha debido, en su opinión, a diversas razones, entre las que destaca el hecho de que los mecanismos de decisión potencian elevados grados de cohesión y solidaridad entre sus miembros. Por ello, considera que el mantenimiento del lazo trasatlántico es una condición prioritaria en toda remodelación futura. Esto obliga a buscar la fórmula que permita compaginar la existencia de la OTAN con las necesidades de la defensa europea. Sería bueno, en su opinión, que Europa reforzara sus capacidades de defensa, para mejorar la movilidad estratégica, la logística, y las necesidades de mando, control e inteligencia. Afirmó el coronel Parente que las diferencias que pudieran surgir entre Estados Unidos y Europa no deberían de afectar nunca a la cohesión del lazo trasatlántico. Esta misma idea recorría las palabras del representante de la Embajada de Estados Unidos, señor Butler, quien llegó a afirmar que la OTAN ha sido la institución de seguridad que ha formado el eje, alrededor del cual casi todas las democracias del continente europeo han ido constituyendo un todo cada vez más coordinado. También resaltó que la entrada de España en la Alianza sirvió para reforzar el enfoque de la misma sobre su flanco sur. En su opinión, la actual ampliación de la OTAN sirve para exportar la estabilidad política y social a esa región. En cualquier caso, serviría para reparar un acto de violencia histórica: la partición de Europa.

En la misma línea de repaso histórico, el Tte. General Muñoz Grandes destacó los cambios de enfoque que acontecieron en las diferentes etapas que siguieron a la Segunda Guerra Mundial. Destacaremos aquí los relativos a la década de 1990, durante la cual se pasó decididamente del concepto de "defensa" al concepto de "seguridad"; las amenazas dejaron de ser concretas, convirtiéndose en difusas y multidireccionales; y se apostó por la multinacionalidad en operaciones 
de paz, con lo que se supera el concepto de alianza cerrada, se refuerza la solidaridad internacional, y se pone de relieve la necesidad de liderazgo; pero, al mismo tiempo, plantea el peligro de que las fuerzas armadas pasen a funcionar como una ONG, y , afirmó el general, conviene no perder la perspectiva y tener claro que su función sigue siendo prepararse para la guerra. Todo ello, en opinión del General Muñoz Grandes, pone de relieve la necesidad de prever estructuras que sustituyan a las que se derrumban, ya que las improvisaciones pueden ser muy peligrosas, y más aún los vacíos, especialmente los ideológicos. Para este conferenciante, la peor consecuencia del 11 de Septiembre fue que todo el entramado construido pareció derrumbarse . En los EE.UU. descubrieron que eran permeables al terrorismo y al crimen organizado, lo que les hizo entrar en una fase de angustia y de DUDAS sobre cómo reaccionar.

Todos los conferenciantes coincidieron en la consideración de que el cambio y modernización de la OTAN era importante, pues de otra forma la Alianza no estaría ajustada a las necesidades del sistema mundial actual, ni sería capaz de cumplir la misión de seguridad para la que existía. Uno de ellos, el profesor Rafael Bardají, citó a Sir. Winston Churchill al comentar que "mejorar es cambiar, ser perfecto es mejorar a menudo". El señor Butler, afirmó que la cumbre de Praga "ha sido el comienzo de un proceso de construcción de la OTAN del futuro". En su opinión, los ataques del 11 de Septiembre no se dirigían solo contra los Estados Unidos, sino contra el mundo occidental y sus valores. Valores que se defienden a través de la Alianza Atlántica. Por ello, la OTAN ha pasado de la defensa territorial de Europa a defender a "Europa, los Estados Unidos y Canadá de fuerzas que pueden atacar desde puntos muy lejanos de este continente". En este sentido, destacó el hecho de que en la operación militar desarrollada en Afganistán, denominada Libertad Duradera, habían participado todos menos dos de los diecinueve aliados de la OTAN.

El profesor R. Bardají señaló el hecho de que la cumbre de Praga originariamente había sido concebida como la cumbre de la segunda ampliación, y había terminado convirtiéndose en la cumbre de la posible transformación de la OTAN.

\section{LAS CUATRO CAPACIDADES OPERATIVAS}

En Praga se tomaron tres acuerdos fundamentales: a) la ampliación, con el ingreso de siete nuevos miembros; b) fomentar y profundizar en las relaciones de amistad y cooperación con Rusia y con otros países del entorno estratégico de la Alianza; y c) dotarse de nuevas capacidades militares que se consideraron necesarios para asegurar la eficacia de la institución. El teniente General Narro, comandante en Jefe del cuartel general de la OTAN en Retamares, explicó detalladamente en qué consistían estos acuerdos.

El apartado relativo a la consolidación de lazos de solidaridad con otros países incluyó varias zonas estratégicas. Por una parte, reforzar el partenariado 
estratégico con Rusia, y el programa de Partenariado para la Paz (PFP) con los países que hoy ocupan el espacio que abarcaba el antiguo Pacto de Varsovia. Respecto al Diálogo Mediterráneo, señaló el general que, en la reunión de Washington se utilizó el término "ampliar", mientras que en Praga se habló de "elevar el grado de importancia". Igualmente, señaló que se habló de reforzar la solidaridad estratégica entre la Alianza y la Unión Europea.

Los cambios más importantes afectaron al ámbito militar. En la cumbre de la OTAN de Praga se ha decidido dotar a la Alianza de nuevas capacidades, en el plano operativo.

Para ello, los Estados Unidos propusieron la creación de una Fuerza de Respuesta Rápida, capaz de desplegar 21.000 tropas, de aire, mar y tierra, dentro o fuera de área, en un plazo de entre siete y treinta días. Debe de tener la capacidad de desplegar cinco mil de esos efectivos en los primeros cinco días. Y sería sostenible hasta un mes. Esta iniciativa sería compatible con el esfuerzo de la Unión Europea de desarrollar la capacidad de desplegar una fuerza de acción rápida de hasta 60.000 tropas, porque la europea está diseñada para funcionar en casos de operaciones de mantenimiento de la paz, mientras que la de la OTAN se considera necesaria para enfrentarse a amenazas concretas. La capacidad operativa de la misma debería ser efectiva para el año 2004, y estar totalmente completada para el 2006.

También se propuso en Praga dotar a la Alianza de una nueva estructura de cuarteles, para asegurar la eficacia del mando en la nueva situación. Está previsto que los Ministros de Defensa de los países miembros se reúnan en Junio de 2003, para aprobar una estructura más flexible y suave. Se ha planteado que esta incluiría tres cuarteles generales conjuntos a nivel operativo, y varios mandos a nivel táctico que se ocupen de las funciones de los componentes de tierra, mar y aire.

El interés de estas transformaciones reside en conseguir dotar a la alianza de mayor operatividad, buscando mejorar las capacidades de despliegue, flexibilidad y capacidad de permanencia. Pero también se toman en consideración un buen número de funciones operativas en tiempo de paz: por ejemplo, la creación de ocho cuarteles generales de entrenamiento, para el control y apoyo de los nuevos miembros. El denominado Compromiso de Capacidades de Praga se centra en cuatro áreas, que se consideran importantes para la actividad futura: a) defensa contra ataques químicos, biológicos, radiológicos y nucleares; b) asegurar una superioridad de mando, comunicaciones e información; c) mejorar la interoperatividad de las fuerzas desplegadas; y d) asegurar la rapidez del despliegue y la capacidad de sostenimiento de las fuerzas.

El general Narro destacó, asimismo, la importancia que se concedió en la cumbre de Praga a la asunción de un mayor nivel de compromiso nacional, a la cooperación multinacional, incluyendo el refuerzo mutuo y a conseguir darle un empujón a la Unión Europea para que desarrolle una mayor capacidad militar. 
La cooperación es fundamental para los temas relativos al terrorismo. También este apartado ha sido reforzado durante la cumbre de Praga. Se han identificado un número de medidas de largo alcance: un concepto militar de defensa anti-terrorista, un plan de acción para mejorar las capacidades que permita controlar consecuencias, defenderse de posibles ataques cibernéticos e incluso preparar una posible emergencia resultante de un fenómeno de desconexión de la OTAN, así como solicitar contribuciones nacionales de elementos críticos de infraestructura, como son los sistemas de información y comunicaciones.

En este sentido, el Sr. Butler hizo hincapié en el hecho de que el invitar a los aliados europeos a participar en mayor medida en el aumento de capacidades de la alianza no equivale necesariamente a pedirles que gasten más, ya que los europeos disponen de capacidades técnicas concretas que pueden aportar, ampliando así los recursos de la Alianza a través de una modificación de los gastos, lo que puede significar un empleo más racionalizado de los mismos, en lugar de su aumento.

Resultó especialmente interesante la reflexión del profesor Rafael Bardají sobre las posibilidades reales de la OTAN, alianza defensiva de tipo militar, de colocar la lucha anti-terrorista en el centro de sus misiones. Usando sus palabras, "el problema es que el propósito es tan bueno como ingenuo". Dado que, en este ámbito, la mejor opción defensiva es la prevención, el protagonismo debería de estar localizado en los servicios de inteligencia y en las unidades policiales. Precisamente, lo que menos se ha desarrollado en la OTAN son las vertientes del intercambio de información e inteligencia. Aunque, evidentemente, podrían desarrollarse. La fase en la que este profesor considera más posible la intervención de la OTAN es en la de "represalia". Afganistán sería en mejor ejemplo de ello.

Por esta razón, el profesor Bardají insistió en la importancia del desnivel existente entre las capacidades militares de Estados Unidos y las de sus aliados europeos. Esto explicaría el hecho de que el aumento de capacidades acordado en Praga se haya reducido a cuatro áreas. En su opinión, esa diferencia no se debe tanto a las diferencias de cantidad de dinero gastado en defensa, sino a que los europeos hace tiempo que decidieron orientar sus gastos de defensa en la dirección de la prevención y la intervención humanitaria. Esto evidenciaría que "los Estados Unidos y Europa no comparten la misma cultura estratégica: mientras que la Europa kantiana se movería en un mundo post-moderno y post-bélico, los Estados Unidos seguirían rigiéndose por principios nacionales, en un mundo hobbesiano en el que el uso de la fuerza no solo es legítimo, sino algo indispensable". Por su parte, el General Muñoz Grandes opinaba que la cumbre de Praga podía ser un acicate para que la Unión Europea quebrase su inmovilismo y su egoísmo en un tema tan trascendental como es el de la seguridad.

Por otra parte, el tema de la lucha antiterrorista ha suscitado el debate sobre la conveniencia de ataques preventivos y anticipatorios. El General Powell aseguró, durante la cumbre, que los ataques preventivos han sido siempre una 
posibilidad en los planes de defensa, mientras que otros participantes aseguraban que su aceptación expresa podía arrastrar al mundo hacia situaciones imposibles de calcular de antemano.

En suma, la cumbre de Praga aparece como un paso adelante en un proceso de transformación de las formas y los medios utilizados hasta ahora por la Alianza Atlántica, que incluye aspectos de transformación de su organización militar, y asimismo incorpora un aspecto muy importante relativo a la relación entre Estados, tanto de sus miembros (que pasarán a ser veinticinco) como con otros que en la actualidad están fuera de ella. Incluyendo esa especie de puente que en la actualidad es la Federación Rusa, con su estatus especial dentro del Consejo OTAN-Rusia. Podríamos cerrar con las palabras del profesor Bardají, quien dijo que "si de verdad la OTAN pasa a ser una caja de herramientas y un facilitador de procedimientos para formar "coalitions of the willing", la OTAN del 2003 y la de 1949 solo compartirán el nombre.Pero puede muy bien que eso no sea mala cosa. 
FUGITIVOS DEL HOGAR

Julio Almeida*

Al hecho claro de que la socialización del niño español se hace hoy más fuera que dentro del hogar, se añade la circunstancia de que no se sale del mismo al final de la adolescencia, como se hacía desde tiempo inmemorial hasta ayer, como se sigue haciendo en muchos países occidentales; quizá (caso estupendo) ni a los veinticinco o treinta, y entonces se cuadra la paradoja: por un lado, el hogar familiar se habita tan poco que las habitaciones pasan a denominarse, han pasado a ser, meros dormitorios; hasta la biblioteca puede llamarse dormitorio; por otro, tan singular socialización, que estorba la autonomía de la personalidad en desarrollo, no les impide a los chicos quedarse en la casa paterna hasta mucho después de la adolescencia. Hijos y padres educados todos en el barullo cotidiano — sobrinos, suegros, etcétera - configuran así un peculiar e inextricable universo que se combina con la vida conversada del bar y con la movida correspondiente. La vividez incomparable de los bares españoles, más numerosos que los de muchos otros países juntos, depende menos de su posible comodidad que de una vida familiar que expulsa tanto como atrae; el bar se nutre en buena medida de la imposible habitabilidad de tales viviendas-dormitorios, de los constantes ruidos de vecinos incapaces de ensimismamiento. Dicen que ahora el paro dificulta y agrava la emancipación de los hijos, pero todo parece indicar que el familismo viene de muy atrás. ${ }^{1}$ Y no se sabe si la disminución del paro, que amontona a la gente en pisos ridículos donde mayormente se conversa a voz en grito y se oye la radio y se ve la televisión en pantalla grande, resolverá el problema que nos ocupa.

* Catedrático de Sociología de EU, Universidad de Córdoba, y miembro de la Asociación Castellano-Manchega de Sociología. 


\section{RADICADOS EN EL HOGAR}

Los datos muestran y demuestran que en las casas españolas viven más personas que en las otras viviendas europeas. «Las estadísticas de alargamiento de la cohabitación entre padres e hijos durante la década de los ochenta, con períodos de dependencia doméstica muy superiores a los europeos... son también un indicador importante del papel de amortiguador social que ha jugado la familia en relación con el choque del paro... $\rangle^{2}$ En otro lugar, en una extensa obra sobre Historia de las mujeres, se recuerda que la cohabitación entre generaciones y fratrías adultas había dejado de ser la regla al final de la Edad Media. Poco después de la segunda Guerra Mundial el baby-boom coincidió con una escasez de viviendas, pero treinta años más tarde disminuye el número de personas en cada casa. «Los hogares en los que no había más de una o dos personas constituían más de la mitad del total de hogares en los países escandinavos, Austria,

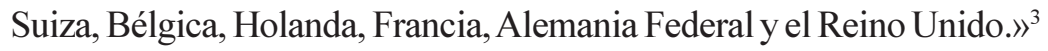

En los países mencionados ha aumentado considerablemente el número de personas que viven solas. A juicio de la socióloga citada, «este aumento de la cantidad de personas que viven solas se debe en gran parte a la ruptura generalizada de la cohabitación entre las generaciones adultas, al crecimiento general de la esperanza de vida y a la mayor longevidad de las mujeres». Pese a las alarmas, la familia se mantiene con buena salud. Ahora bien, ya no se trata de aquella gran familia tradicional que educaba a sus muchos hijos en parte en la calle, sin coches hasta hace pocos decenios; en plan piara, como decía un chico educado entre diez hermanos. En la mayoría de los países europeos y en Estados Unidos, los hijos piensan irse del hogar paterno al terminar la escuela secundaria, que coincide con el final de la adolescencia; aunque sigan estudiando, ya se consideran adultos que pueden y deben contribuir a subvenir a sus necesidades. ${ }^{4}$

La peculiaridad española no estriba sólo en el hecho de que ahora parece difícil encontrar empleo: es que muchos jóvenes consideran natural seguir viviendo donde los nacieron, parasitando sine die a sus mayores. No se les ocurre que podrían abandonar el nido familiar y embridar su existencia. Se diría que van a quedarse en la adolescencia para siempre. Tan lejos de la mentalidad europea como de la española preindustrial, nuestros jóvenes actuales (tan numerosos, por otra parte) ignoran la ley natural que obliga a separarse de la teta nutricia y a vivir autónomos; se hallan tan bien instalados bajo el techo en que nacieron, que parecen dispuestos a no dejarlo jamás.

En El niño difícil (1930) calculaba Alfred Adler que del 50 al 60 por ciento de todos los niños han sido acostumbrados a depender de otras personas. «Esta falta de independencia se manifiesta a lo largo de su vida: todo es difícil para ellos; no confían en su propia capacidad.» Setenta años después tendríamos que hacer otros cálculos. Sin duda los jóvenes españoles actuales dependen demasiado de sus progenitores y temen echarse a volar por su cuenta. 


\section{FUGITIVOS DEL HOGAR}

Pero hay más, el otro lado de la cuestión. No se trata sólo del hecho calculable de que en las casas españolas viven o intentan convivir más personas que en las de otros países; casi nadie ignora que suele tratarse de pisos tan pequeños que difícilmente se puede vivir en ellos. Por lo general son viviendas sin habitaciones habitables, y su conversión en dormitorios no es ningún desliz semántico; a decir verdad, esos habitáculos se habitan poco porque no dan para mucho. A veces los periódicos dan la imposible noticia de que en tal sitio de veinte metros cuadrados viven veinte personas; pero querrán decir que pernoctan. Por lo tanto, cuando se habla de la formidable socia(bi)lidad de los españoles, hay que matizar. Los frecuentadísimos bares, en efecto, no se nutren sólo de su posible comodidad o atractivo, como sucede en otros países; se llenan también, así como calles y paseos, porque el minúsculo hogar carece de sitio y de posibilidad de sosiego y expulsa a sus inquilinos, que vienen y van constantemente. Al exponer estas ideas en clase, una alumna comentó con inteligencia: En realidad, estamos en casa de visita.

Ahora se habla mucho de fracaso escolar, pero yo me pregunto cómo puede estudiar un chico en viviendas que tienen tan pocos metros cuadrados por persona. ¿Cómo trabajar con tanta gente y tantos decibelios: en el piso propio, en el de arriba o abajo, en el de al lado? Ya los constructores se cuidan de que la estructura del edificio sea endeble, de que techos y tabiques aíslen poco o nada; el amor al ruido y los horarios extravagantes los ponemos nosotros, o nos los impone algún vecino.

Vemos que las apariencias engañan; como dice Berger, las cosas no son lo que parecen. La calle viva, los innúmeros bares y cafés casi siempre abiertos y llenos de gente, creo que dependen precisamente de la fuga de quienes no disponen en su casa de espacio y calma suficientes; por lo demás, evidentemente, nuestra extroversión y nuestra verborrea arrancan de una socialización brutal que nos habitúa desde niños a convivir y a conversar sin medida. ¿Leer El Quijote? Es un libro demasiado largo, contestan sin rebozo algunos pobrecitos estudiantes.

Hablamos de la vida cotidiana, pero todo está conexionado. Cuando, en 1924, reflexionaba Ortega en el centenario del nacimiento de Kant, ya advirtió nuestro filósofo que el meridional vive «condenado al barullo de la gran plazuela cósmica y no se hallará jamás verdaderamente solo». No es retórica. La situación del español es inversa de la del alemán, para quien el yo está siempre consigo; su ser es un serpara-sí, el Fürsichsein hegeliano. «Durante quince años de cátedra he podido adquirir la más amplia experiencia de la enorme dificultad con que una cabeza española llega a comprender este concepto [de autoconciencia, o conciencia de sí, Selbstbewusstsein]. En cambio, me sorprendió muchas veces en los seminarios filosóficos alemanes la facilidad con que el principiante penetra dentro de él. Era el pato recién nacido que se lanza recto a la laguna, su elemento.» ${ }^{5}$ Poco después, en ¿Qué es Filosofía?, precisará que la idea de subjetividad, principio básico de la 
Edad Moderna, es tan fácil y obvia para los hombres del norte como incomprensible para nosotros; y esta incomprensión «es una de las razones por las cuales los pueblos mediterráneos no han sido nunca plenamente modernos». Verdaderamente, la exaltación del yo tiene largas consecuencias. ¿Puede alguien pensar todavía que la historia es un proceso natural?

\section{LA VIDA APARENTE}

Con la Reforma, los europeos protestantes arribaron a la convicción de que cada hombre responde de su vida ante el sumo Hacedor sin subterfugios colectivistas y debe por tanto dedicarse a un trabajo determinado, aunque sea humilde. En cambio el español, que hacía la guerra divinal por casi todo el mundo, confiaba más en Dios y se cuidaba menos de los negocios cismundanos; menos de las cosas que de la honra de las personas, más del ser que de los resultados; quality frente a performance, en el esquema de variables de Parsons. Cuando se convive con españoles o con norteamericanos o europeos del norte, se ve que los primeros, más atentos a la presión ajena, parecen figurantes de una vida social (máximamente en Sevilla) y afectan un nivel de vida visible superior al de aquéllos, mayor en todo caso que el nivel efectivo. Esto lo ha observado Juan José Linz, pero lo hemos visto cuantos hemos vivido fuera. A su modo lo había señalado Gracián, al promediar el siglo XVII, en El Criticón: como primera en todo lo malo, la soberbia cogió la delantera y se acomodó en España. "Allí vive y reina con todos sus aliados, la Estimación propia, el Desprecio ajeno, el querer mandarlo todo y servir a nadie, hacer del don Diego y vengo de los godos, el Lucir, el Campear, el Alabarse, el Hablar mucho, alto y hueco, la Gravedad, el Fausto, el Brío, con todo género de Presunción, y todo esto desde el noble hasta el más plebeyo.»

Los extranjeros que nos visitan no pueden entender las arcanas razones que llevan a nuestras mujeres a acicalarse tanto un día cualquiera para salir de casa a comprar tomates. Como ellos y ellas van a lo suyo, en el sentido meliorativo (el ocuparse de sus propios asuntos, que los ingleses parece que aprendieron en el Quijote, Dios mío, ¿será verdad?), ignoran que nosotros vamos al teatro de la calle para vernos y hablar de lo divino y de lo humano; ignoran sobre todo nuestro pavor al qué dirán, un qué dirán que en el rigor de los términos es una presionante opinión que decimos todos sin inhibiciones. El espectáculo de una calle o un paseo de Córdoba, por ejemplo, tiene poco que ver con la mundana religiosidad de esos protestantes lanzados con fiera circunspección a su clase de piano, a su hora de inglés, a su deporte favorito. Ellos no han venido al mundo a charlar sin tasa. Esas diferencias, por consiguiente, tienen menos que ver con el clima que con la historia, y si una muchacha andaluza quiere aprender a tocar el piano será censurada como ninguna sueca puede imaginar, y la marearán constantemente con una pregunta desoladora: ¿Y eso, para qué? 
En fin, ensimismarse es un verbo espléndido que sólo tenemos en español, pero ¿quién sabe por qué? Aquí es más difícil ensimismarse que en Francia o en Dinamarca. En 1926 Unamuno escribía a su amigo Jean Cassou: «¿Por qué no lanza usted en francés un s'ensoimêmer?» Probablemente no necesitan conjugarlo.

\section{A MODO DE CONCLUSIÓN. UNA HABITACIÓN PROPIA}

La historia no concluye nunca, pero he de cerrar esta breve nota sobre la paradójica vida fugitiva de tantos españoles. Peor que la situación del varón fue durante milenios la de la mujer, menos libre y autónoma. Pues bien, al final de los años veinte, cuando las mujeres occidentales empezaban a estudiar y a votar y a entrar en la escena pública, un grupo de feministas preguntaron a Virginia Woolf por las mujeres y la novela. ¿Por qué había tan pocas mujeres escritoras? Y la novelista contestó con sensatez que para poder escribir novelas, el quid latente de la pregunta, una mujer debe tener dinero y una habitación propia.

Una habitación propia —room inglesa grande, no dormitorio minúsculo, quizá para varios - es la condición material necesaria; las otras condiciones (talento y dedicación) difícilmente pueden desarrollarse sin aquélla. Lo que en 1929 pedía una mujer de la elite de Bloomsbury, podemos generalizarlo hoy y suponer que sin un espacio y un tiempo propios, hay poco que hacer y se busca la salvación en la huida. ¿A qué obedecen si no esas extrañísimas movidas que fascinan a nuestros jóvenes? No se refugiarían en ellas si tuvieran verdaderos hogares, es decir, espaciosos y amables.

Cuando murió Luis Rosales, Julián Marías se preguntaba por qué entre todo lo que escribió el poeta prefería La casa encendida y agregaba: «La vida humana transcurre en una casa; en muchas, que en definitiva son la misma.» Yo creo que estas palabras del maestro podrían discutirse en el sentido que vengo señalando aquí. En círculos altos o cultivados, sí; en otros países, sin duda; pero para una infinidad de españoles, la vida transcurre más fuera que dentro de casa, y esto desde párvulos, cuyo horario — a diferencia de lo que se hace por ahí- se iguala bárbaramente con el de los mayores: con el de los niños de doce años, con el de su maestra. Para muchos, vivir en su hogar es muy difícil, porque faltan metros o sobra gente, o las dos cosas. Muchos hispanos podrían suscribir las palabras del protagonista de Don Segundo Sombra: «La calle fue mi paraíso, la casa mi tortura.»

El inglés afirma, por lo visto, que su casa es su castillo, My home is my castle. Yo he pensado en ocasiones que no pocos españoles, fugados desde luego de su hogar, dirán más bien: Mi casa es mi dormitorio. 


\section{NOTAS}

${ }^{1}$ Véase mi término "Familismo", en: UÑA, O. y HERNÁNDEZ, A. (2003): Diccionario de sociología, ESIC, Madrid.

${ }^{2}$ ZALDIVAR, C.A. y CASTELLS, M., (1992): España fin de siglo, Madrid, pp, 126-327.

"LEFAUCHEUR,N.; "Maternidad, familia, Estado", en DUBY,G. Y PERROT, M. ( 1993): Historia de las mujeres en Occidente, 5 vols., Madrid, p.444

${ }^{4}$ Para Estados Unidos, puede verse VARENNE, H., "Love and liberty. La familia americana contemporánea", en la obra colectiva dirigida por BURGUIÈRE y otros (1988): Historia de la familia, 2 vols., II,Madrid, pp. 425-448

"Reflexiones de centenario. 1724-1924". OC, IV, p.35 ss. 